\title{
reviscafuences
}

ISSN: 1575-7072 | e-ISSN: 2172-7775

Páginas: 203-211

Recibido: 2020-02-19

Revisado: 2020-04-01

Aceptado: 2020-05-25

Preprint: 2020-07-15

Publicación Final: 2020-09-15 www.revistascientificas.us.es/index.php/fuentes/index

DOI: https://doi.org/10.12795/revistafuentes.2020.v22.i2.07

\section{Disociaciones entre discurso pedagógico y prácticas lectoras en futuros profesores: pistas para la formación docente}

\section{Dissociations between pedagogical discourse and reading practices in future teachers: tips for teacher training}

\author{
(iD) Carla Muñoz \\ Universidad Católica del Maule (Chile) \\ (iD) Constanza Lobos \\ Universidad Alberto Hurtado (Chile) \\ iD Jorge Valenzuela \\ Universidad Católica del Maule (Chile)
}

\begin{abstract}
Resumen
Las creencias y representaciones operan sobre la acción, por lo que es posible pensar que el tipo de mediaciones que realizará el profesor en el aula dependerán de su bagaje lector, sus concepciones sobre la lectura y de la consecuente relación que establezca con ella. El objetivo de este estudio fue caracterizar las creencias de profesores en formación en torno a la lectura, así como su relación con la formación personal y profesional. Para ello, se realizaron cuatro grupos focales con estudiantes de pedagogía en dos regiones del país $(n=23)$, en los cuales se indagó sobre sus creencias sobre la lectura en general y como objeto de enseñanza, así como su relación con la lectura personal y profesional. Los discursos fueron objeto de un análisis de contenido asistido por Nvivo-11. Los resultados muestran una definición no unitaria de la lectura, en donde predominan aspectos positivos como su valor y beneficios para el desarrollo, no obstante, se observa una disociación entre esta declaración y sus prácticas. Por un lado, estos estudiantes afirman leer por gusto, pero no demuestran conocimiento literario ni expresan preferencias. Por otro lado, identifican dificultades para leer a causa de su excesiva carga docente. A nivel profesional, aunque reconocen su rol como mediadores de lectura, advierten que no es una tarea exclusiva del docente. Se discuten las implicancias de estas disociaciones para la formación de profesores y de nuevos lectores.
\end{abstract}

\begin{abstract}
Beliefs and representations influence action, so it is not unfeasible that teacher interventions made in the classroom depend on their reading background and their conceptions of reading. Thus, the objective of this study was to characterize the beliefs of pre-service teachers in training regarding reading, as well as their relationship with personal learning and professional development. To this end, four focus groups were conducted with pre-service teachers in two regions of Chile $(n=23)$, in which their beliefs about reading in general and as an aspect of teaching were investigated, as well as their own relationships with personal and professional reading. The results show dissonant definitions, where in spite of participants' predominantly positive regard for reading, such as its value and benefits for development, there is a disconnect between such affirmations and the practices of participants. On the one hand, participants claim to read for pleasure, but do not demonstrate literary knowledge, nor do they express preferences. On the other hand, they identify difficulties in reading because of their excessive teaching loads. At a professional level, even though participants acknowledge their role as reading mediators, they recognize that this is not a task exclusive to teachers. This paper discusses the implications of these findings for preservice teachers and for new readers.
\end{abstract}

\section{Palabras clave / Keywords}

Lectura; formación de docentes; creencias; hábito de lectura; formación de lectores.

Reading, reader training; beliefs; reading habits, teacher training. 


\section{Introducción}

No cabe duda que cuando pensamos en la Escuela, pensamos también en el proceso de alfabetización. Sin embargo, junto con enseñar a decodificar y comprender textos, es imprescindible que el profesor motive a la lectura, al disfrute y compromiso con la lectura independiente, fuera y dentro de la escuela (Gambrell, 2015). En efecto, la institución escolar es el lugar en donde formalmente ingresamos a la cultura escrita. Así, una de las tareas de la Escuela es la formación de nuevos lectores. En este contexto, la figura del maestro es entendida como la de un mediador en la cultura, de cuyo bagaje lector y de la relación que establezca con la lectura derivarán mediaciones más o menos efectivas (Daisey, 2009; Powell-Brown, 2003).

La investigación ha mostrado, desde diversas aproximaciones, la existencia de una relación significativa entre el hábito lector del docente y el nivel de motivación e implicación de sus estudiantes con la lectura (McKool \& Gespass, 2009; Morrison \& Wlodarczyk, 2009). En el ámbito anglosajón, esta problemática ha sido denominada como "Efecto Pedro" (Applegate et al., 2014) aludiendo a la expresión -basada en la parábola bíblica- "nadie da lo que no posee". En dicho sentido, la figura utilizada en este contexto releva el mandato social del docente en tanto modelo lector para sus alumnos. En otras palabras, si el maestro no posee o no desarrolla el gusto por la lectura, difícilmente logrará que sus estudiantes lo desarrollen. Bajo esta premisa, nuestro estudio ha buscado indagar en la relación del futuro profesor con la lectura a nivel personal y profesional y cómo sus creencias juegan un rol en la representación de este objeto en el espacio escolar, de sus prácticas personales de lectura y de su relación con este objeto cultural.

Respecto de la lectura, la investigación muestra que las creencias juegan un rol tanto en el aprendizaje como en la enseñanza (Hong \& Nguyen, 2019). Si bien en la literatura es posible advertir un uso variado y no siempre consistente del concepto creencias, no obstante es posible consensuar ciertos aspectos (Fives \& Buehl, 2012). En efecto, se trataría de construcciones mentales de base subjetiva, que actúan como filtros acerca del mundo. Asimismo, aunque no existe consenso entre los autores respecto de su naturaleza implícita o explícita, sí se reconoce que en cuanto a estructura su organización estaría dada en forma de sistema, aunque no necesariamente en una forma lógica (Pajares, 1992), ello explicaría, en parte, que las personas pudieran sostener creencias incompatibles o inconsistentes (Van Driel, Bulte, \& Verloop, 2007).

En el ámbito educacional, la profusa investigación sobre las creencias de los profesores da cuenta del interés por comprender cómo estas podrían estar moldeando la acción de los docentes (Cf. Ashton, 2015). En dichos estudios se observa que las creencias de los profesores están fuertemente influenciadas por tres elementos: los estudiantes, el contenido y sus creencias personales derivadas de la experiencia (Fives \& Buehl, 2012). Si bien los estudios sobre las creencias de los profesores de lenguaje en torno a la enseñanza de la lengua tienen una larga data (véase, por ejemplo, la revisión realizada por Borg, 2015); solo de manera reciente se han desarrollado investigaciones sobre las creencias del profesor acerca de la lectura y las relaciones que se establecen con ésta en su vida personal y profesional (Cf. Maggioni, Fox, \& Alexander, 2015). Si como la investigación lo ha demostrado, la actuación pedagógica del profesor se nutre de su saber, sus creencias y pensamientos; se hace relevante no solo estudiar qué saben los profesores de primaria y de educación inicial sobre lenguaje, sino también qué creen y piensan sobre la lectura y la formación de nuevos lectores. Respecto de sus saberes, aunque los Estándares Orientadores para carreras de Educación Inicial en Chile (MINEDUC, 2012) plantean como deseable tanto el conocimiento y selección de obras de literatura infantil nacional e internacional de acuerdo con "criterios de calidad gráfica, de formato y contenido"; estudios muestran que sólo $53 \%$ de las carreras de educación inicial en Chile tiene alguna asignatura de literatura infantil, y que, cuando este sistema literario se incluye en la formación, suele predominar la revisión de corpus de obras clásicas por sobre la lectura y análisis de la literatura infantil contemporánea (Molina, 2014). Asimismo, investigaciones en diversas latitudes muestran la precariedad de hábitos lectores, conocimientos literarios y manejo del lenguaje especializado en futuros maestros primarios (Deleuze, 2009; Larrañaga \& Yubero, 2005); todos aspectos que influyen en sus actuaciones pedagógicas. En efecto, se ha observado que aquellos profesores que poseen hábitos de lectura consolidados son más proclives a emplear buenas prácticas, compartiendo sus gustos literarios en el aula, promoviendo la discusión literaria o la lectura en voz alta (Burgess, Sargent, Smith, Hill, \& Morrison, 2011; McKool \& Gespass, 2009).

El presente estudio, se inscribe dentro de un enfoque referido a cognición docente $(B o r g, 2015)$ y se centrará específicamente en las creencias de profesores en formación en torno a la lectura, así como su relación con la formación personal y profesional. 


\section{Metodología}

Este estudio exploratorio de carácter cualitativo, se inserta dentro de un proyecto mayor sobre el profesor como lector cuyo objetivo fue caracterizar las creencias de profesores en formación en torno a la lectura, así como la relación de estas lecturas con su formación personal y profesional. Para estos efectos, se optó por la utilización del Grupo Focal como técnica de recolección de datos (Krueger \& Casey, 2009), asegurándonos que tanto en el diseño como en la ejecución de la investigación, se consideraran los criterios de calidad sugeridos por la COREQ checklist (Booth et al., 2014), así como el resguardo ético de los participantes (Steneck, Mayer, \& Anderson, 2010).

\subsection{Participantes}

Participaron estudiantes de pedagogía, de ciclo terminal en Educación Inicial y Primaria, de ambos sexos (69\% mujeres) provenientes de tres universidades en las regiones de Valparaíso y Araucanía ( $n=23)$, y cuya edad promedio fue de 27 años ( $s d=5.8$ ).

Fueron considerados como criterios de inclusión, estudiantes que pertenecieran al ciclo terminal de formación pedagógica; ello, bajo la premisa de que estudiantes de años superiores tendrían representaciones y creencias sobre la lectura consolidadas y, a la vez, tributarias de la formación inicial docente.

La aproximación a los participantes se realizó por medio de visitas en aulas universitarias donde se realizó una invitación formal de participación a los estudiantes. Quienes demostraron interés en participar, fueron citados a una reunión formal para estos efectos. Cada sesión del grupo focal fue precedida de una introducción, por parte de uno de los investigadores, en donde se contextualizó el motivo de la invitación a participar en la actividad y en donde cada uno de los estudiantes aceptó formalmente participar por medio de la firma de un protocolo de consentimiento informado. Los Grupos focales se realizaron en un espacio de tres meses y el número final realizado para este estudio $(n=4)$ estuvo determinado por un criterio de saturación teórica de la información (Ardila \& Rueda, 2013; Flick, 2009).

\subsection{Instrumentos/Materiales}

Se optó por el Grupo focal como estrategia de recolección de datos dado el potencial para identificar, entre otros, las actitudes, creencias y experiencias de los participantes a través de la interacción dialógica del grupo (Gibbs, 1997). Esta técnica posibilita la expresión de un rango más amplio de reflexiones por parte de los participantes que aquellas que podrían haber sido establecidas por el investigador en un formato menos dinámico (Callaghan, 2014).

Los grupos focales fueron guiados a través de un protocolo de preguntas que indagaba sobre tres aspectos concretos: a) sus creencias respecto de la lectura en general y b) la lectura como objeto de enseñanza, así como c) su relación con la lectura personal y profesional. La discusión estuvo a cargo de dos miembros del equipo ambos con postgrados en el área de ciencias sociales y experiencia en investigación, quienes actuaron como moderador y observador, respectivamente. Ninguno de ellos conocía a los participantes ni poseía vínculo previo con éstos. Los datos fueron registrados en audio y video, y luego, transcritos y revisados por los miembros del equipo. El guion de grupo focal, así como la grilla de codificación se encuentran disponibles como anexos a este artículo.

\subsection{Análisis de datos}

Los grupos focales fueron objeto de un análisis de contenido (Erlingsson \& Brysiewicz, 2017; Mayring, 2015) asistido por Nvivo 11. La codificación, de carácter mixto, fue realizada en dos etapas. En un primer momento, se elaboró un sistema de códigos, a partir de una definición consensuada por el equipo en torno a los tópicos de interés de investigación, lo que permitió agrupar la información contenida en los textos analizados. Esta codificación fue implementada en una primera fase y luego de un análisis parcial del material, esta fue reajustada en nodos temáticos relacionados con los ejes estructurantes que guiaron la conversación de los grupos focales. A partir de la revisión de categorías preestablecidas por el equipo y la discusión respecto a su ajuste, se realizó el análisis del corpus. A partir de este proceso recursivo se construyeron nuevas categorías, mientras que otras se fueron fusionando o eliminando según su pertinencia teórica y práctica. Finalmente, el sistema de códigos definitivo permitió analizar los cuatro grupos focales, abarcando los contenidos más relevantes que emergieron durante la conversación. La información producida se organizó 
en cuatro grandes dimensiones: 1) Creencias sobre la lectura, 2) El docente como mediador, 3) El docente como lector y 4) Relación profesional con la lectura: estrategias para fomentar la lectura.

\section{Resultados 1}

\subsection{Creencias sobre la lectura}

Respecto de la lectura, las creencias de estos futuros profesores se articularon en torno a ideas y nociones cercanas a la formación inicial docente. La noción más aludida en el discurso de los entrevistados refirió a la lectura como proceso de comprensión. Para ambos educadores, la lectura es comprensión -oral o escrita- de textos: "Cuando comprende, según yo, porque si no sería decodificar" (F1p3). "Cuando [el niño] tiene una comprensión de algún texto y no necesariamente tiene que saber leer él directamente, sino que puede uno leerle y él escucha lo que uno está diciendo y si lo comprende se considera una forma de lectura" (F1p7).

En este sentido, los estudiantes de pedagogía señalan que las obras literarias serían una forma de expresión, siendo la lectura un diálogo constante entre el lector, el autor y el propio libro o texto y donde el rol del lector es comprender aquello que se trata de comunicar. A un nivel más general y menos mencionado por los entrevistados, indican una relación entre lectura con cultura, historia y acceso a la información. En efecto, la lectura y en particular los textos serían un medio que permite el acceso a temáticas de diverso orden, a hechos históricos, a nuevo vocabulario y a conocimiento específico de los textos (como el tipo de lenguaje, géneros, entre otros).

Consultados por la definición de lectura, se desprenden una serie de atributos -en su mayoría positivos- que propiciarían en las personas esta actividad. Las referencias aluden en mayor medida al desarrollo de habilidades cognitivas, permitiendo la adquisición de conocimientos, el desarrollo del pensamiento (crítico, espacial, histórico, integral) y una mejor comprensión del mundo. A su vez, los estudiantes entrevistados señalan que la lectura permite identificar el desarrollo y la conexión con las propias emociones y experiencias personales y de otros. En la misma línea, otro atributo que se asocia a la lectura es la cualidad de propiciar la configuración de la identidad en el individuo. En efecto, los participantes aseguran que el sujeto puede encontrarse consigo mismo a través de las lecturas que realiza, pero que esta tiene también el potencial de acercar a otros, gracias a las experiencias vicarias a las que se exponen mediante esta práctica.

Sí, creo que la lectura es como un encuentro, [...]. En la lectura uno se dispone a esos encuentros, ya sea con los personajes, con lo que nos dice el libro, (...) pero después con los años hay cierto tipo de lectura con la que uno se identifica y dentro de esta construcción de la identidad, (...) la lectura pasa a ser, por lo menos para mi, como una forma de encontrarte, de encontrar paz, de encontrar conocimiento, encontrarme con otros, encontrarme con ideas, con explicaciones, con un montón de cosas que ese encuentro me entrega, de saberes que -en el fondo- ese encuentro me entrega (F2p3).

Junto con lo anterior, se le atribuye a la lectura la condición de ser un hábito de carácter voluntario que debe ser motivado por el interés individual, es decir, esta actividad no debiese tener un carácter obligatorio. A la vez, se advierte en el discurso una percepción instaurada de la lectura como una actividad funcional, sobre todo en contextos académicos:

lo importante es que tiene que ser voluntario, porque uno quiere hacerlo, porque si no, después vas perdiendo ese gusto por la lectura y se le agarra la fobia. Como que está instaurado [en la escuela] de que es lectura por obligación (F1p2).

Finalmente, la mayoría de los participantes en sus discursos, declaran la importancia de leer reivindicando la libertad del lector de poder elegir el género que más les guste ("que lean manga, si quieren"). De este modo, para estos futuros docentes la práctica lectora sería fundamental con prescindencia del contenido o género.

${ }_{1}$ Para efectos de identificación y resguardando la identidad de los participantes se han codificado las intervenciones con el siguiente código: Focus $\mathrm{n}^{\circ}$, participante ํㅡ․ 


\subsection{La lectura como objeto de enseñanza: El docente como mediador}

Respecto del vínculo entre labor docente y lectura, la idea del docente como mediador es transversal entre estos estudiantes de Pedagogía. En este sentido, existe conciencia del mandato social impuesto al docente en tanto modelo para sus estudiantes y su rol en el proceso de transmisión del gusto por la lectura y la enseñanza de un hábito lector:

Bueno, nosotras como educadoras, tenemos que ser modelo de referencia para los niños, y que nosotras también le demostremos que, nosotras igual leemos, que los espacios que se den para que ellos observen los libros y nosotros igual lo hagamos para que ellos puedan ver la motivación que tenemos nosotros (F4p4).

No obstante, también se reconoce un papel fundamental del contexto familiar en que se desarrollan los niños y que, según los entrevistados, es una base para consolidar el gusto por la lectura. En esta línea, rescatan la importancia de factores como el nivel socioeconómico, el capital cultural y las posibilidades de otros soportes de acceso a la lectura, en especial el soporte digital, como algunos de los elementos más mencionados que facilitan u obstaculizan el desarrollo lector. Así, la labor de enseñanza y transmisión del gusto por la lectura sería también responsabilidad del entorno familiar. De manera complementaria a esta idea, recogimos algunos discursos presentes en estos futuros docentes donde se observa todavía una visión parcializada de la formación de nuevos lectores como tarea particular del profesor de lenguaje: "Es que, dado el sistema educativo chileno, el [profesor] de lenguaje es el que se debe encargar de esa tarea" (F2p3).

En consonancia con ello, señalan otros actores que tendrían la labor de mediar la lectura, reconociendo el carácter social de esta. Así, en la sociedad existirían diversos mediadores dependiendo de la situación, del contexto y del tipo de relación con la lectura que se pretenda transmitir: "(...) tampoco se puede desconocer el rol del Estado, de las universidades, de las bibliotecas públicas, (...), pero también el rol de la familia". (F3p3).

Ahora bien, respecto a la importancia que la lectura tendría para la labor docente, los entrevistados señalaron que un docente lector podría contagiar su interés por la lectura y, al mismo tiempo, otorgar a sus estudiantes mayores posibilidades de lectura, incorporando los intereses de los alumnos, a partir de su conocimiento en el tema. En este punto es donde radicaría la importancia del rol del docente para la transmisión del gusto y hábitos de lectura. Además, la lectura facilitaría enseñar y permitir el acceso al conocimiento para los estudiantes y, al mismo tiempo, posibilitaría una relación más cercana entre profesor-estudiantes, debido a que la lectura propiciaría conocer el contexto de origen e intereses de los niños.

No obstante, los docentes entrevistados reconocen una serie de limitaciones para su rol de mediadores en la lectura los cuales obstaculizarían la transmisión del gusto por la lectura hacia sus estudiantes. Por una parte, se culpa a la rigidez del curriculum que limitaría al docente, por ejemplo, dando un listado fijo de obras para lectura en la escuela; impidiendo la programación de otros textos y actividades que fomenten la lectura. Por otra parte, el acceso material a textos u obras literarias en la población general es indicado como un factor importante en la motivación por la lectura. Por ende, la falta de acceso -asociado al costo del libro- sería también una limitación para fomentar el gusto por la lectura en los estudiantes.

\subsection{Relación del futuro maestro con la lectura personal y profesional: El docente como lector}

En contraposición con la importancia atribuida a la lectura presente en la conversación con los participantes de los grupos focales, éstos reconocen que, tanto para los profesores en general como para los estudiantes de pedagogía la lectura no sería un hábito frecuente en su vida cotidiana. En efecto, a pesar de la importancia señalada por nuestros participantes acerca de la relación entre lectura y docencia, así como a las potencialidades de la lectura para su labor docente los entrevistados reconocen que sus compañeros y colegas no leen o dedican poco tiempo a esta actividad. En este punto es que resulta fundamental preguntarse por los factores que podrían obstaculizar esta práctica.

Un factor que aparece de manera consistente en todos los grupos focales es la carencia de tiempo para la lectura. La extensión de su jornada laboral, más allá de las clases lectivas en la Escuela, iría en desmedro del tiempo que disponen para actividades de carácter personal:

Porque la carga laboral es demasiada (...) el trabajo no se termina en el colegio, uno tiene que llegar a la casa a hacer material, hacer pruebas, corregir cosas y te dan ¿Cuánto, las 10 de la 
noche? Y de ahí a leer por placer... o tú dices "ah, ya tengo el fin de semana" pero resulta que el fin de semana tengo que hacer más cosas para el colegio, más cosas para esto, entonces como que el tiempo personal se va agotando o se va reduciendo en ese aspecto. Entonces, como de marzo a diciembre, yo creo que no. Enero y febrero sí (F1p2).

Del mismo modo, nuestros participantes insisten en que el poco tiempo para la lectura personal estaría destinado a temas de interés para ellos y, por ende, no siempre se destina a leer textos respecto de su labor docente.

De manera emergente y aunque mencionado en menor medida, resulta importante destacar que algunos entrevistados reconocen dificultades para leer. Dentro de éstas, se señalan dificultades de comprensión lectora y de concentración, que limitarían la cantidad de lecturas del docente.

Ante la pregunta sobre la relevancia de ser un buen lector para trabajar como docente, los participantes destacan que un profesor lector podría motivar a sus estudiantes, mediante la búsqueda de estrategias para fomentar el gusto por la lectura, como la creación de espacios de lectura con los estudiantes y la incorporación de temáticas o autores que sean del interés de los niños y niñas. Es interesante notar en este punto que, aunque los entrevistados indican la importancia de la lectura para establecer vínculos sociales y afectivos con los estudiantes, no existen alusiones personales a la lectura. En otras palabras, ninguno de nuestros participantes recoge su propia experiencia lectora como insumo para tomar decisiones sobre la lectura sugerida para sus estudiantes. No se observa en ninguno de los grupos focales la referencia explícita a obras o autores; ninguno de los participantes refiere a su propia experiencia como lector. Sin embargo, al mismo tiempo nuestros entrevistados afirman que un profesor-lector estaría dotado de conocimientos y habilidades que facilitarían su trabajo de enseñanza de la lengua en el aula:

Porque si uno no comprende siendo profesor ¿cómo le enseñas a tus estudiantes a comprender? ¿cómo le enseñas también, en el caso de redactar, si tú no sabes redactar? ¿cómo le enseñas a ellos redactar? O si no tienes buena ortografía ¿Cómo les enseñas ortografía? (F1p3).

\subsection{Estrategias para fomentar la lectura}

Otra temática que fue comentada con interés por los estudiantes de pedagogía durante los Grupos focales fueron aquellas estrategias que permitiesen fomentar o incentivar esta práctica, tanto en el papel de estudiantes y lectores como en su rol de mediadores y docentes. Como futuros docentes, los entrevistados plantean que la lectura funcional estaría instalada en la sociedad y en el sistema escolar. Para contrarrestar esto, sugieren fomentar la lectura en la escuela a partir de la motivación. Desde esa discusión se plantean estrategias tales como experiencias compartidas de lectura entre profesores y estudiantes (debido a que el profesor sería un referente para sus estudiantes), incorporar temáticas de interés de los estudiantes en las lecturas escolares, generar aprendizajes significativos a través de la lectura y la conexión que ella tendría con las emociones y experiencias personales. Todo ello generaría interés en los estudiantes.

Esta misma idea se replica en el caso de los propios entrevistados en su rol de estudiantes, pero en un nivel diferente. Si bien muchos se declaran lectores o interesados por la lectura, expresan que faltan espacios de lectura, de debate y reflexión en las universidades que fomenten esta actividad, para así ayudar a formar a "docentes buenos lectores" que potencien sus competencias en tanto mediadores.

\section{Discusión}

El objetivo del presente estudio fue caracterizar las creencias de profesores en formación en torno a la lectura, así como la relación de ésta con la formación personal y profesional del futuro docente.

Una primera conclusión que emerge de los resultados obtenidos en este estudio es la clara disociación que se observa en los discursos de estos futuros profesores entre las dimensiones personal y profesional de la lectura. Esto es, en tanto lectores y mediadores de la lectura. En efecto, observamos un discurso socialmente aprendido en donde queda claramente establecido el rol del profesor en tanto enseñante de lengua y mediador de lectura. Sin embargo, se observa una precariedad del discurso asociado a la lectura en la esfera personal. No hay referencia a obras, autores, géneros o estilos preferidos; algo similar a lo referido en estudios internacionales (Émery-Bruneau, 2011). Ante la evidencia de esta precariedad de lecturas en la esfera personal, las razones esgrimidas son, de preferencia, la carencia de tiempo para esta actividad o las dificultades de lectura. Aunque la primera es una razón que coincide con estudios en diferentes latitudes 
(Borg, 2009; Huang, 2017; Skaar, Elvebakk, \& Nilssen, 2018), merecería la pena seguir indagando sobre las dificultades específicas de lectura en la edad adulta y en específico, en la población de futuros docentes, dado su rol clave en la formación de nuevos lectores.

Asimismo, en la dimensión profesional también observamos una clara disociación: si bien la lectura es referida como condición para ser un mejor docente, a la hora de reflejarlo en sus propias prácticas, ésta aparece claramente disminuida. En consecuencia, es difícil hablar propiamente de "profesores lectores" (Commeyras, Bisplinghoff, \& Olson, 2003) y más bien estaríamos en presencia de lo que ha sido catalogado como "falsos lectores" (Larrañaga \& Yubero, 2005), pues aunque algunos de los participantes declaran ser lectores y desear más espacios de lectura, esas lecturas están desvinculadas de su desarrollo académico o profesional. En efecto, destaca la creencia sostenida por estos futuros docentes sobre la lectura como una herramienta o medio para alcanzar objetivos que dan cuenta de un foco preferente de carácter funcional. Dicho reduccionismo se expresa también en la forma en que se relacionan los futuros docentes con los textos. Desde su rol mediador, para ellos los textos se constituyen de manera preferente en medios para enseñar habilidades (ortografía, redacción, ampliación de vocabulario), haciendo alusión -nuevamente- a la lectura desde un enfoque funcional. Desde esta perspectiva, prima en sus discursos la labor del docente de enseñar las habilidades de leer y comprender, dejando de lado la dimensión fruitiva de la lectura.

No obstante lo anterior, aparece el interés como eje estructurante del hábito, algo ya reportado por la literatura (Guthrie, Hoa, Wigfield, Tonks, \& Perencevich, 2006). La consideración del interés como algo exclusivamente intrínseco, puede suponer un dilema para estos futuros pedagogos, condicionando sus prácticas pedagógicas a los "intereses de los niños". Esta mirada simplista del interés (cf. Renninger \& Hidi, 2016) deja en evidencia, a la vez, una carencia de marcos orientadores de la acción pedagógica y, de manera consecuente, una reflexión más profunda de parte de estos futuros pedagogos; dos aspectos claves a los que atender en la formación inicial de docentes.

Las creencias que fue posible pesquisar en este estudio dan cuenta de que la lectura es conceptualizada siempre con valencia positiva y que es una habilidad que requiere ser enseñada (decodificación y comprensión). Esta visión positiva, deja de lado sin embargo lo fruitivo. De esta manera, el gusto por leer solo emerge como un criterio clave para validar la lectura cuando es voluntaria, pero independiente de su valor literario ("que los niños lean lo que quieran" [con tal de que lean]). Así, la práctica lectora es validada solo cuando no es obligatoria. Dicho de otro modo, parece ser justificable para estos futuros docentes que no exista una práctica lectora sostenida cuando no media la voluntariedad en la lectura. Por lo anterior, no se trataría sólo de un factor de autodeterminación (Ryan \& Deci, 2017), sino una manera de justificar la carencia de una práctica considerada positiva y que es extensiva no sólo a la lectura de ocio, sino que también a la lectura académica. Esta "criminalización" de la lectura sugerida o impuesta de manera externa plantea fuertes desafíos a la formación de futuros profesores, pues ¿cómo se motiva a los estudiantes a leer si no existen instancias intencionadas explícitamente en donde se practique la lectura? Las causas de esta situación también se constituyen en un desafío para los investigadores. ¿Será esa obligatoriedad de la lectura en sus propias experiencias como estudiantes lo que detona un desinterés por la lectura a nivel personal? Creemos fundamental profundizar en estos aspectos en futuras investigaciones.

Junto con estas creencias en torno a la dimensión individual de estos futuros profesores, aparece una serie de creencias en la dimensión profesional. La primera, tiene que ver con la responsabilidad docente (Lauermann, 2014) ya que habría una tendencia a des-responsabilizar al profesor de primaria de la formación de lectores, y atribuir dicha tarea específicamente al profesor de lenguaje. Esta rigidez en la concepción del modelo de formación es preocupante, si se piensa que se trata de futuros profesores cuya responsabilidad formal pasa por la iniciación de la lectura y la responsabilidad social de asumir una tarea que la familia, sobre todo en contextos más desfavorecidos, no puede asumir. También se observa una distinción entre futuras educadoras de nivel inicial y docentes de primaria. Las primeras enfatizan su rol como fomentadoras del gusto, mientras que los segundos insisten en su rol como enseñantes. Esta falta de articulación también es preocupante para una adecuada transición entre los niveles y evidencia una incomprensión del desarrollo lector como un continuo. Este aspecto debe ser revisado en la formación inicial de docentes enfatizando un adecuado dominio no solo de las didácticas específicas a cada ciclo, sino también la deseable práctica colegiada que potencie las transiciones educativas entre niveles.

\section{Conclusiones}

A partir de este estudio, hemos podido indagar en las creencias que poseen futuros profesores en torno a la lectura, así como la relación que sostienen con ésta a nivel personal y profesional. 
La conclusión central de este estudio es la confirmación de una clara disociación entre creencias y prácticas de lectura de este grupo de estudiantes que confirma lo observado en estudios de distintas latitudes (Dengler, 2018; Skaar et al., 2018) y que nos lleva a cuestionar el currículum de formación docente, así como el currículum escolar. A la par de un pobre acervo literario, estos futuros docentes muestran poca o nula referencia a prácticas sostenidas de lectura personal. En efecto, sus positivas creencias respecto de los beneficios de leer y ser lector contradicen de manera palmaria sus prácticas personales y profesionales. Asimismo, nuestros resultados muestran que esta falta de prácticas lectoras intencionadas por la formación docente limita la posibilidad de que estos jóvenes puedan desplegar todo el potencial pedagógico para mediar la formación y desarrollo de nuevos lectores. Sabiendo la influencia que poseen las creencias en la acción, es imperativo intervenir en la modificación de las creencias sobre la lectura que permitan modificar los hábitos lectores de los futuros docentes, los que esperamos enriquezcan el trabajo de mediación lectora del educador en el aula.

Una vía de intervención que parece auspiciosa es la consideración de la lectura en estos futuros docentes como una vía de configuración identitaria. Experiencias llevadas a cabo en Estados Unidos con clubes docentes de lectura (Cf. "Teachers as Readers" en Commeyras et al., 2003; Cremin, Mottram, Collins, Powell, \& Safford, 2009), muestran cómo la inclusión de la lectura en la vida del docente puede provocar cambios en la manera en que este se posiciona en tanto sujeto lector-enseñante. De alguna forma, esto nos lleva a cuestionar los modelos de formación docente centrados en el aprendizaje de didácticas específicas de la lectura y la escritura, que prescinden de esta dimensión identitaria como una primera etapa fundamental para establecer trayectorias de lectura que enriquezcan la vida lectora personal del futuro enseñante. Experiencias como estas, -no sólo de lectura literaria, sino también disciplinar- han reportado beneficios no solo a nivel motivacional por la lectura, sino también se ha observado un enriquecimiento personal del conocimiento literario, así como en la autoimagen del profesor como lector y en sus actuaciones pedagógicas. Esto refuerza la idea de apoyar dispositivos y experiencias que doten de autoeficacia al nuevo profesor, quien a su vez se sentirá más seguro para compartir en aula sus preferencias literarias, así como su punto de vista, estimulando la discusión literaria en aula, una práctica efectiva pero poco utilizada por los docentes. Estas experiencias de lectura compartida podrían ser incluidas como parte nuclear del currículum de formación en tanto experiencias compartidas para futuros docentes de educación inicial y primaria.

\section{Apoyos}

Esta investigación ha sido financiada por la Comisión Nacional de Investigación en Ciencia y Tecnología (Conicyt-Chile) a través del Proyecto Fondecyt Regular 1170779.

\section{Referencias}

Applegate, A. J., Applegate, M. D., Mercantini, M. A., McGeehan, C. M., Cobb, J. B., DeBoy, J. R., . . Lewinski, K. E. (2014). The Peter Effect revisited: Reading habits and attitudes of college students. Literacy Research and Instruction, 53(3), 188-204. doi:https://doi.org/10.1080/19388071.2014.898719

Ardila, E., \& Rueda, J. (2013). La saturación teórica en la teoría fundamentada: su de-limitación en el análisis de trayectorias de vida de víctimas del desplazamiento forzado en Colombia. Revista colombiana de sociología, 36(2), 93114. Retrieved from https://revistas.unal.edu.co/index.php/recs/article/view/41641/43310

Ashton, P. T. (2015). Historical overview and theoretical perspectives of research on teachers' beliefs. In H. Fives \& M. Gregoire (Eds.), International handbook of research on teachers' beliefs (pp. 31-47). New York: Routledge.

Booth, A., Hannes, K., Harden, A., Noyes, J., Harris, J., \& Tong, A. (2014). COREQ (Consolidated Criteria for Reporting Qualitative Studies). In D. Moher, D. G. Altman, K. F. Schulz, I. Simera, \& E. Wager (Eds.), Guidelines for Reporting Health Research: A User's Manual (pp. 214-226): John Wiley \& Sons, Ltd.

Borg, S. (2009). English language teachers' conceptions of research. Applied linguistics, 30(3), 358-388.

doi:10.1093/applin/amp007

Borg, S. (2015). Teacher cognition and language education: Research and practice. London: Bloomsbury Publishing. Burgess, S. R., Sargent, S., Smith, M., Hill, N., \& Morrison, S. (2011). Teachers' leisure reading habits and knowledge of children's books: do they relate to the teaching practices of elementary school teachers? Reading Improvement, 48(2), 88.

Callaghan, J. (2014). Focus Groups Introduction. New York: Springer Science+Business Media.

Commeyras, M., Bisplinghoff, B. S., \& Olson, J. (Eds.). (2003). Teachers as Readers: Perspectives on the Importance of Reading in Teachers' Classrooms and Lives. Newark: Delaware: ERIC.

Cremin, T., Mottram, M., Collins, F., Powell, S., \& Safford, K. (2009). Teachers as readers: building communities of readers. Literacy, 43(1), 11-19. doi:10.1111/j.1741-4369.2009.00515.x

Daisey, P. (2009). The Reading Experiences and Beliefs of Secondary Pre-service. Reading Horizons, 49(2), 167-190.

Retrieved from https://scholarworks.wmich.edu/reading_horizons/vol49/iss2/6 
Deleuze, G. (2009). Futurs instituteurs et faibles lecteurs: réalité et/ou fatalité. Enjeux, 74, 93-100.

Dengler, K. A. (2018). Aliterate Pre-Service Teachers' Reading Histories: An Exploratory Multiple Case Study. State University of New York.

Émery-Bruneau, J. (2011). Former des «sujets-lecteurs-enseignants»: une responsabilité collective pour des actions didactiques réfléchies. Québec français(163), 79-81.

Erlingsson, C., \& Brysiewicz, P. (2017). A hands-on guide to doing content analysis. African Journal of Emergency Medicine, 7(3), 93-99. doi:10.1016/j.afjem.2017.08.001

Fives, H., \& Buehl, M. M. (2012). Spring cleaning for the "messy" construct of teachers' beliefs: What are they? Which have been examined? What can they tell us. APA educational psychology handbook, 2, 471-499. doi:10.1037/13274-019 Flick, U. (2009). An introduction to qualitative research. London: Sage.

Gambrell, L. B. (2015). Getting Students Hooked on the Reading Habit. Reading Teacher, 69(3), 259-263. doi:10.1002/trtr.1423

Gibbs, A. (1997). Focus groups. Social research update, (19). Retrieved from http://www.soc.surrey.ac.uk/sru/sru19.html Guthrie, J., Hoa, L., Wigfield, A., Tonks, S., \& Perencevich, K. (2006). From Spark to Fire: Can Situational Reading Interest Lead to Long-Term Reading Motivation? Reading Research and Instruction, 45(2), 27.

doi:10.1080/19388070609558444

Hong, T. D., \& Nguyen, H. B. (2019). Teacher beliefs and practices of scaffolding students' reading comprehension through questionning at pre-reading stage. European Journal of Foreign Language Teaching, 4(2), 72-92. doi:10.5281/zenodo.3270743

Huang, S. (2017). Reading Practices of Pre-Service Teachers in the United States. Reading Psychology, 38(6), 580-603. doi:10.1080/02702711.2017.1310160

Krueger, R. A., \& Casey, M. A. (2009). Focus groups: A practical guide for applied research. USA: Sage.

Larrañaga, E., \& Yubero, S. (2005). El hábito lector como actitud. El origen de la categoría de "falsos lectores". Ocnos:

Revista de estudios sobre lectura(1), 43-60. doi:10.18239/ocnos_2005.01.04

Lauermann, F. (2014). Teacher responsibility from the teacher's perspective. International Journal of Educational Research, 65, 75-89. doi:10.1016/j.ijer.2013.09.005

Maggioni, L., Fox, E., \& Alexander, P. A. (2015). Beliefs about reading, text, and learning from text. In H. Fives \& M. Gregoire (Eds.), International Handbook of research on teacher's belief. (pp. 353-369). Abingdon: Routledge. Mayring, P. (2015). Qualitative content analysis: Theoretical background and procedures Approaches to qualitative research in mathematics education (pp. 365-380): Springer.

McKool, S. S., \& Gespass, S. (2009). Does Johnny's Reading Teacher Love to Read? How Teachers' Personal Reading Habits Affect Instructional Practices. Literacy Research and Instruction, 48(3), 264-276.

doi:10.1080/19388070802443700

MINEDUC. (2012). Estándares orientadores para carreras de educación parvularia. Estándares pedagógicos y disciplinarios. Santiago: Ministerio de Educación. Recuperado de http://portales.mineduc.cl/usuarios/cpeip/File/librosestandaresvale/libroparvulariafinal.pdf.

Molina, V. (2014). Literatura infantil en la carrera de educación de Párvulos. Un estudio sobre su presencia en Chile. Tesis presentada para la obtención del grado de Máster en Libros y Literatura Infantil y Juvenil. Universidad Autónoma de Barcelona.

Morrison, V., \& Wlodarczyk, L. (2009). Revisiting read-aloud: Instructional strategies that encourage students' engagement with texts. The Reading Teacher, 63(2), 110-118. doi:10.1598/rt.63.2.2

Pajares, M. F. (1992). Teacher's beliefs and educational research: Cleaning up a messy construct. Review of Educational Research, 62(3), 307-332. doi:10.2307/1170741

Powell-Brown, A. (2003). Can You Be a Teacher of Literacy If You Don't Love to Read? Journal of Adolescent \& Adult Literacy, 47(4), 284-288. Retrieved from http://www.jstor.org/stable/40014774

Renninger, K. A., \& Hidi, S. (2016). The power of interest for motivation and engagement. New York: Routledge. Ryan, R. M., \& Deci, E. L. (2017). Self-Determination Theory. Basic Psychological Needs in Motivation, Development, and Wellness. New York: Guilford Press.

Skaar, H., Elvebakk, L., \& Nilssen, J. H. (2018). Literature in decline? Differences in pre-service and in-service primary school teachers' reading experiences. Teaching and Teacher Education, 69, 312-323. doi:10.1016/j.tate.2017.10.019 Steneck, N., Mayer, T., \& Anderson, M. (2010). Singapore statement on research integrity. Principles and Responsibilities for Research Worldwide. Retrieved from https://wcrif.org/guidance/singapore-statement. Van Driel, J. H., Bulte, A. M. W., \& Verloop, N. (2007). The relationships between teachers' general beliefs about teaching and learning and their domain specific curricular beliefs. Learning and Instruction, 17(2), 156-171. doi:10.1016/j.learninstruc.2007.01.010 